\title{
BMJ Open Effect of computerised, knowledge- based, clinical decision support systems on patient-reported and clinical outcomes of patients with chronic disease managed in primary care settings: a systematic review
}

Marie line El Asmar (D) , ${ }^{1}$ Kanika I Dharmayat, ${ }^{2}$ Antonio J Vallejo-Vaz,, ${ }^{3,4,5}$ Ryan Irwin, ${ }^{6}$ Nikolaos Mastellos ${ }^{7}$

To cite: El Asmar Mline, Dharmayat KI, Vallejo-Vaz AJ, et al. Effect of computerised, knowledge-based, clinical decision support systems on patient-reported and clinical outcomes of patients with chronic disease managed in primary care settings: a systematic review. BMJ Open 2021;11:e054659. doi:10.1136/ bmjopen-2021-054659

- Prepublication history and additional supplemental material for this paper are available online. To view these files, please visit the journal online (http://dx.doi.org/10.1136/ bmjopen-2021-054659).

$\mathrm{RI}$ and NM contributed equally.

Received 23 June 2021 Accepted 22 November 2021

Check for updates

(C) Author(s) (or their employer(s)) 2021. Re-use permitted under CC BY-NC. No commercial re-use. See rights and permissions. Published by BMJ.

For numbered affiliations see end of article.

Correspondence to Dr Marie line El Asmar; me719@ic.ac.uk

\section{ABSTRACT}

Objectives Chronic diseases are the leading cause of disability globally. Most chronic disease management occurs in primary care with outcomes varying across primary care providers. Computerised clinical decision support systems (CDSS) have been shown to positively affect clinician behaviour by improving adherence to clinical guidelines. This study provides a summary of the available evidence on the effect of CDSS embedded in electronic health records on patient-reported and clinical outcomes of adult patients with chronic disease managed in primary care.

Design and eligibility criteria Systematic review, including randomised controlled trials (RCTs), cluster RCTs, quasi-RCTs, interrupted time series and controlled beforeand-after studies, assessing the effect of CDSS (vs usual care) on patient-reported or clinical outcomes of adult patients with selected common chronic diseases (asthma, chronic obstructive pulmonary disease, heart failure, myocardial ischaemia, hypertension, diabetes mellitus, hyperlipidaemia, arthritis and osteoporosis) managed in primary care.

Data sources Medline, Embase, CENTRAL, Scopus, Health Management Information Consortium and trial register clinicaltrials. gov were searched from inception to 24 June 2020.

Data extraction and synthesis Screening, data extraction and quality assessment were performed by two reviewers independently. The Cochrane risk of bias tool was used for quality appraisal.

Results From 5430 articles, 8 studies met the inclusion criteria. Studies were heterogeneous in population characteristics, intervention components and outcome measurements and focused on diabetes, asthma, hyperlipidaemia and hypertension. Most outcomes were clinical with one study reporting on patient-reported outcomes. Quality of the evidence was impacted by methodological biases of studies.

Conclusions There is inconclusive evidence in support of CDSS. A firm inference on the intervention effect was not possible due to methodological biases and study
Strengths and limitations of this study

- In contrast to other reviews, this review focuses on interventions involving clinical decision support systems (CDSS) that are knowledge-based, are embedded within electronic health record systems, provide electronic alerts and are used by physicians in primary care.

- The electronic search strategy was piloted, and hand searching of reference lists of previous reviews permitted a sufficient level of confidence that all relevant articles were included.

- Some limitations arose related to studies being of 1-year duration on average, making it difficult to deduce long-term effects on patient outcomes.

- The scope of the review was restricted to nine chronic diseases; however, the selection included the most common chronic diseases; yet, there were no eligible studies in five of them.

- The included studies were of low-to-moderate quality of evidence, and heterogeneity among the included studies limited the ability to conduct a meta-analysis.

heterogeneity. Further research is needed to provide evidence on the intervention effect and the interplay between healthcare setting features, CDSS characteristics and implementation processes.

PROSPERO registration number CRD42020218184.

\section{INTRODUCTION}

Non-communicable chronic diseases are the leading cause of disability and mortality worldwide, affecting one in three adults globally. ${ }^{1}$ Rising healthcare expenditure because of chronic disease prevalence has led to an ever-growing demand for effective primary care services for the long-term management of chronic conditions. ${ }^{2-4}$ Considering that 
health systems have mainly adapted to providing periodic care for acute problems, an even greater need to achieve efficient, comprehensive and coordinated long-term care persists. ${ }^{5}$ Models such as the chronic care model have been proposed to improve the quality of care in chronic care systems. ${ }^{6}$ This model recommends 'decision support' as one of six applicable changes in primary care settings that promote better patient outcomes, if successfully implemented.$^{6-8}$ Within this and other models of primary care and chronic condition management, clinicians are encouraged to provide patient care according to evidence-based clinical practice guidelines (CPGs) to support improved patient outcomes. ${ }^{9}{ }^{10}$ Given the frequent updates to CPGs resulting from rapid advancements in medical knowledge, a major challenge is the maintenance of clinicians' knowledge of evidence-based CPGs. ${ }^{10}$ Consequently, clinicians' adherence to CPGs may be variable and frequently insufficient, which may result in suboptimal disease management outcomes. ${ }^{1011}$

Clinical decision support is an umbrella term that includes various care structures, processes and tools. ${ }^{12}$ Clinical decision support systems (CDSS) range from non-computerised to computerised systems and basic to advanced systems. ${ }^{12}{ }^{13}$ Currently, most attention is driven toward computerised advanced CDSS, which perform complex decision-making functions from electronic health record (EHR) patient data. ${ }^{12}$ Such systems are termed knowledge-based, as they use software algorithms to generate actionable patient-specific outputs in the form of recommendations to the practitioner using conditional (IF-THEN) rule statements derived from CPGs. ${ }^{145}$ They also differ in their technical and clinical interface, whether passive (providing user-initiated prompts to generate clinical advice, eg, by clicking buttons) or active (automatic system-initiated prompts). ${ }^{12} 1617$ CDSS also differ in system-user communication, which could be of consulting nature (ie, provide appropriate 'nextsteps' clinical recommendations) or of critiquing nature (verify decisions taken by practitioners). ${ }^{12}{ }^{16}$ Given the differences in interfaces, the scope of this review includes computerised, knowledge-based CDSS embedded in EHRs that are of either passive or active, consulting or critiquing, nature.

Although CDSS are ubiquitous, few have been successfully adopted. ${ }^{18}$ Previous reviews appraised the efficacy of different types of CDSS in primary care, presenting a greater consensus on the useful role of CDSS on practitioner-related outcomes such as guideline adherence. ${ }^{19-23}$ However, the impact of CDSS on patient outcomes remains largely uncertain. ${ }^{19-23}$ Bryan et al demonstrated a positive association between the use of CDSS in primary care and patient outcomes. ${ }^{23}$ However, a need to further evaluate the effect of CDSS on patient outcomes was identified, particularly in primary care and specifically for chronic disease management. The novel focus of this review is in the combined focus on the effect of computerised, knowledge-based, CDSS and their effect on patient-reported and clinical outcomes of patients and with chronic disease and managed in primary care settings. This particular focus was chosen to inform more specific and efficacious implementation strategies in primary care management of chronic disease and attract the industry's consideration of CDSS use in primary care settings, which a more generalised focus on multiple care settings, multiple diseases beyond just chronic disease or lack of focus on patient-reported outcomes may not achieve. There remains a need to assess the capabilities of CDSS in distinctive clinical professional areas and disease entities, particularly on patient outcomes. ${ }^{18} \mathrm{~A}$ recent review on EHR-embedded CDSS focused on process delivery (practitioner outcomes) and was not limited to primary care settings and chronic conditions. ${ }^{24}$ Despite obvious heterogeneity, clinically meaningful improvements in care processes were shown. ${ }^{24}$ However, it was unclear under what clinical circumstances these improvements could be replicated in every clinical context to affect clinical outcomes presumptively. ${ }^{24}$

In this systematic review, patient outcomes refer to quantifiable clinical biomarkers such as glycated haemoglobin $(\mathrm{HbA1c})$ and low-density lipoprotein cholesterol (LDL-C) levels, clinical outcomes such as mortality and morbidity, and patient-reported outcomes, such as healthrelated quality of life. To the best of our knowledge, and in the view of rapid upgrades in the world of CDSS, no review exists solely on the effect of CDSS embedded within EHRs on patient outcomes for patients with chronic disease managed in primary care. This review aims to fill this knowledge gap by collating all the available evidence on this classification of CDSS.

\section{METHODS}

\section{Study design and eligibility criteria}

A systematic review of the existing literature was conducted following the Preferred Reporting Items for Systematic Reviews and Meta-Analyses (PRISMA) guidelines. ${ }^{25} 26$ The research question, inclusion and exclusion criteria were defined using the PICOS framework. ${ }^{27}$ Nine common chronic diseases were selected as part of the inclusion criteria, following a review by Reynolds et $a l^{28}$ namely asthma, chronic obstructive pulmonary disease (COPD), heart failure, myocardial ischaemia, hypertension, type 2 diabetes mellitus (T2DM), hyperlipidaemia, arthritis and osteoporosis. Studies reporting on patientrelated and clinical outcomes managed by primary care clinicians using CDSS-EHR compared with usual care were included. Studies involving participants $<18$ years of age, multidisciplinary (non-clinician) system users, and other varieties of CDSS (not linked with EHR) were excluded. A detailed view of the criteria is provided in online supplemental table 1 .

Figure 1 depicts an analytic framework of the intervention adapted from a framework published by Lobach et $a l^{22}$ The framework provides a theoretical context for possible features that could underpin the success, or lack of success, of a complex intervention such as 


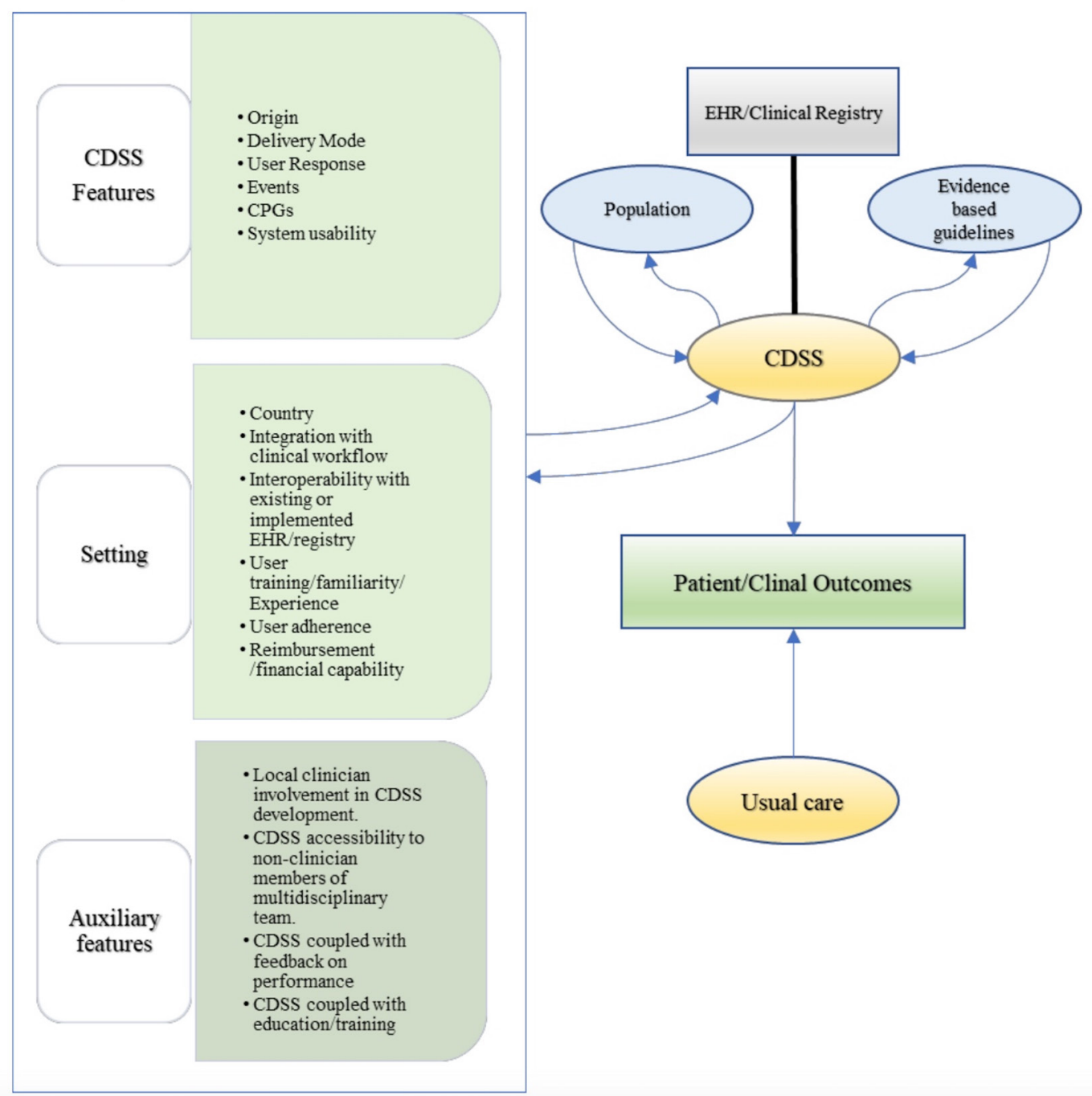

Figure 1 Analytic framework. CDSS, clinical decision support system; CPGs, clinical practice guidelines; EHR, electronic health record.

implementing CDSS in a primary care setting. The framework guided the extraction of relevant data from studies to inform the narrative synthesis.

\section{Search strategy}

Electronic databases Medline, Embase, Scopus, Cochrane Central Register of Controlled Trials (CENTRAL) and Health Management Information Consortium, and one clinical trial registry (clinicaltrials.gov), were searched from inception to 24 June 2020. Cochrane reviews were screened for relevant search terms and subject headings $(\mathrm{MeSH})$ terms under the following categories 'registries supported by CDSS' AND 'General Practice/Primary care' OR each of the nine chronic diseases combined with AND 'Asthma', 'heart failure/myocardial ischaemia', 'hypertension', 'diabetes mellitus', 'hyperlipidaemia', 'COPD', 'arthritis', 'osteoporosis' (online supplemental tables 2 and 3). The search was limited to articles in English.

Terms for patient-reported outcome measures (PROM) were excluded. Existing PROM filters are not comprehensive, and the use of those filters in the search strategy could increase the risk of missing relevant studies. ${ }^{29}$ However, we manually screened papers to identify any 'patient-reported outcomes' measured in studies. PROM may be described in various ways; thus, attempting to list specific patient outcomes for each entity could introduce bias and lead to missing relevant studies. Previous systematic reviews around CDSS in primary care were also searched for potentially eligible studies. ${ }^{19-21} 2324$

\section{Study screening and selection}

Search results were exported to RefWorks, then screened using Covidence. After removal of duplicates, screening was conducted by two reviewers independently (MLEA and KID). Disagreements were resolved by consensus within the review group. After an initial screening of titles and abstracts, the full text of potentially relevant articles was assessed.

\section{Data extraction}

A data extraction form was adapted using the 'Cochrane data collection form for intervention reviews: randomised 
controlled trials (RCTs) and non-RCTs' and aided by Lobach $e t a l$ s data abstraction guidance. ${ }^{30} 31$ The form was piloted and two reviewers (MLEA and KID) completed data extraction independently.

\section{Quality assessment of individual studies}

Risk of bias (RoB) was assessed using the Cochrane RoB tool, ${ }^{32}{ }^{33}$ by two reviewers independently (MLEA and KID). The methodological RoB was assessed per the following elements in RCTs: selection bias, performance bias, detection bias, attrition bias, reporting bias and other biases. ${ }^{33} 34$

Quasi-RCTs were reported as high risk for random sequence generation. Cluster RCTs were assessed for an additional element 'selective recruitment of cluster participants'. All judgements were made with guidance from the Cochrane Handbook and the guidelines of the Cochrane Consumers and Communication Review Group. ${ }^{33-35}$

Studies were considered as high risk if they scored 'high' or 'unclear' for either random sequence generation or allocation concealment, based on the rising evidence that these two elements are particularly significant sources of bias. ${ }^{34}$ A summary 'RoB' figure was generated for all risk assessments of elements with a narrative commentary for each study. Additionally, a detailed adapted table was generated to visualise subelements that fit under each element in the Cochrane RoB tool. ${ }^{34}$

\section{Quality assessment of outcomes}

The Grading of Recommendations, Assessment, Development and Evaluations (GRADE) tool was used to assess the quality of outcomes from individual studies. ${ }^{36}$ The tool involves five elements: RoB, inconsistency, indirectness, imprecision and publication bias. The tool is primarily used for meta-analyses; however, it can be adapted for use in narrative reviews. Study outcomes were appraised, ranked as high, moderate, low or very low and justifications were provided, in line with Cochrane guidance. ${ }^{37}$

\section{Data synthesis}

Treatment effect was reported as ORs with 95\% CIs and $\mathrm{p}$ values for dichotomous outcomes, where available. Continuous data were presented as mean differences with 95\% CIs and $p$ values.

Heterogeneity in the results was anticipated due to the wide spectrum of possible patient-related outcomes reported by the studies. Methodological heterogeneity was assessed using Cochrane RoB tool. Heterogeneity in included studies varied in terms of intervention components, population characteristics and outcome measurements; thus, conducting a meta-analysis was deemed not appropriate by the four-person author group.

\section{Patient and public involvement}

No patients were involved.
RESULTS

The search identified 5430 articles. Following removal of duplicates, 4498 articles remained for screening by title and abstract. Of these, 266 articles were selected for full-text review and 10 articles met the inclusion criteria. Among them, two articles were published protocols of included articles; thus, eight different studies were finally included. The detailed search results are presented in the PRISMA flowchart (figure 2).

\section{Study characteristics}

Characteristics of included studies are presented in table 1. Included studies comprised of patients in four disease areas: T2DM, asthma, hyperlipidaemia and hypertension. ${ }^{38-45}$ No studies on COPD, heart failure, myocardial ischaemia, arthritis and osteoporosis were identified. Six studies were cluster RCTs, one study was parallelgroup RCT and one was quasi-RCT of stepped-wedge design. Most studies were conducted in the USA, and three studies took place in the UK, Spain and Belgium. Studies, both among the disease conditions and within each disease entity, differed appreciably in participant covariates, setting attributes and CDSS characteristics. These factors, compounded with the heterogeneity of the studies (as described in the Methods section) suggested that the reported outcomes were not fit for statistical pooling; hence, these factors justified not conducting a meta-analysis. Follow-up ranged from 6 months to 1.5 years. No study incorporated financial reimbursement before the implementation of interventions. Further details are presented in table 1.

\section{CDSS characteristics}

CDSS interventions were characterised based on their origin (commercial/local), delivery mode (user or system-initiated), user response (optional/mandatory), events (system functions), coupled EHR and auxiliary features (local clinician involvement in system development process, system accessibility to other team members, provision of performance feedback or coupled with user education). Additional information such as user training, user adherence and reported usability was recorded. Details are provided in table 2.

\section{RoB and quality of the evidence}

Online supplemental tables 4 and 5 provide risk assessments details. Overall, the included studies were impacted by RoB, mainly due to incomplete outcome data and uncertainty on blinding of outcome assessment and personnel. In most studies, it was unclear if outcome assessors were blinded. Additionally, blinding of physicians (CDSS users) was not possible across all studies, as they were the intended system users. Most studies reported substantial withdrawal rates due to poor follow-up. These limitations resulted in analysing subgroups (rather than the initially intended full cohorts) in some trials; hence, most studies were considered of high risk for incomplete outcome data. 


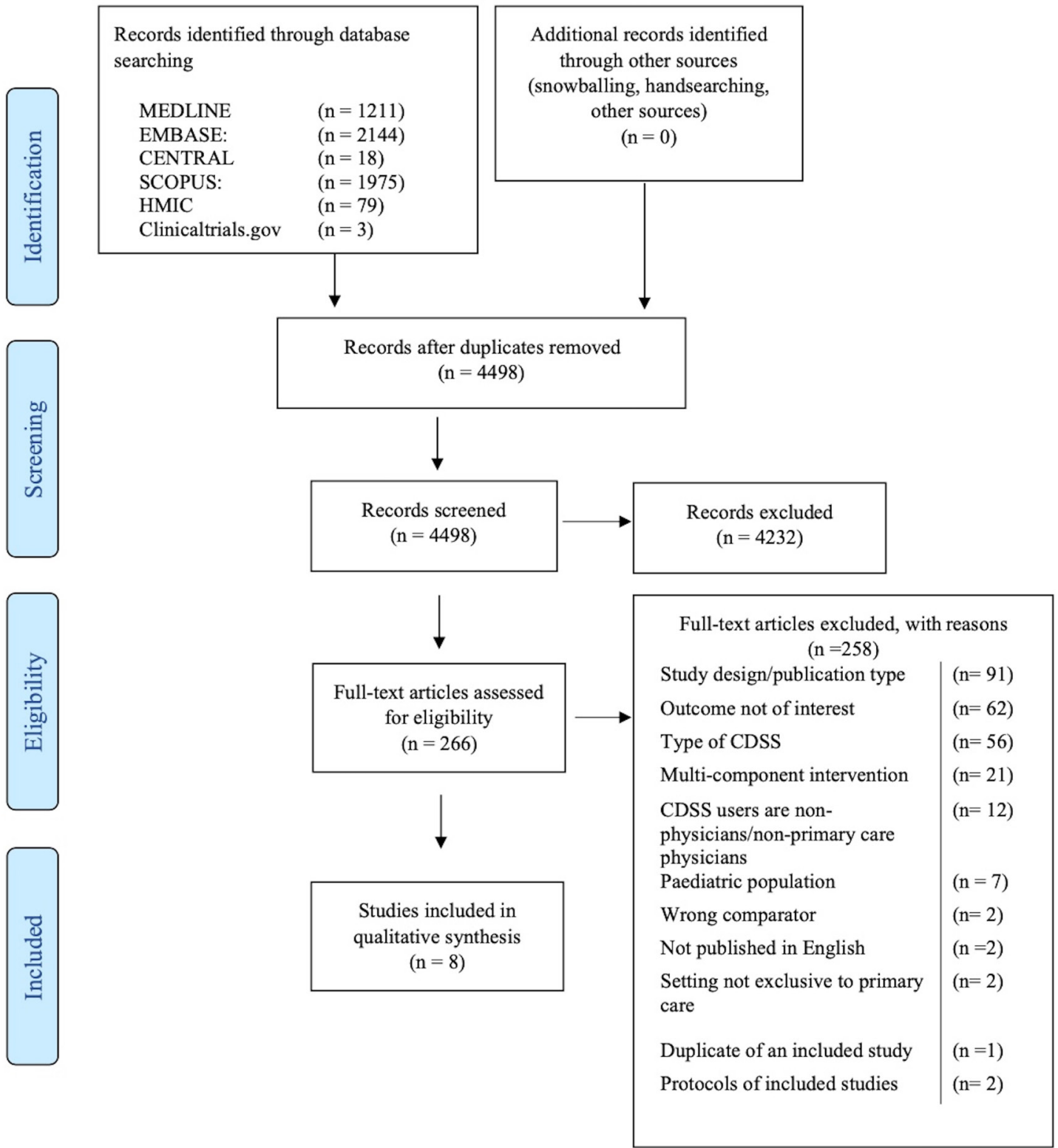

Figure 2 PRISMA flowchart detailing the systematic search results. CDSS, clinical decision support systems; CENTRAL, Cochrane Central Register of Controlled Trials; HMIC, Health Management Information Consortium; PRISMA, Preferred Reporting Items for Systematic Reviews and Meta-Analyses.

Overall, quality of the evidence was rated as either moderate or low, with the most impact resulting from methodological risks of bias within studies. Online supplemental table 6 presents the quality of evidence evaluated using GRADE, with reasons stated for quality downgrading.

\section{Narrative synthesis}

\section{Diabetes mellitus}

Three studies assessed the effect of CDSS on clinical and patient-reported outcomes in patients with T2DM. ${ }^{38-40}$

Gill et al retrieved slight, yet statistically significant, reduced HbAlc and LDL-C with the CDSS intervention compared with the control group, with a small betweengroup difference in HbA1c and LDL-C levels of $0.12 \%$ and $3.57 \mathrm{mg} / \mathrm{dL}$, respectively (table 3). ${ }^{39}$ The reported reductions in HbAlc and LDL-C were clinically small; however, statistically, the intervention group had 52\% higher odds of achieving personalised HbAlc levels, $56 \%$ higher odds of achieving HbAlc $<7.0 \%$ and $34 \%$ higher odds of achieving an LDL-C $<100 \mathrm{mg} / \mathrm{dL}$ compared with usual care patients over 1 year of follow-up. Nevertheless, compared with the control group, the intervention group had better-controlled baseline levels of HbAlc and LDL-C. The study by Gill et al involved system-initiated 'push' CDSS, which were integrated into EHRs already operating at participating practices. Reported limitations were of EHR-CDSS interoperability difficulties, which resulted in missing patient lab values. ${ }^{39}$ Other missing lab values also resulted from patients not returning for lab tests. In view of these limitations, a subset of randomised 

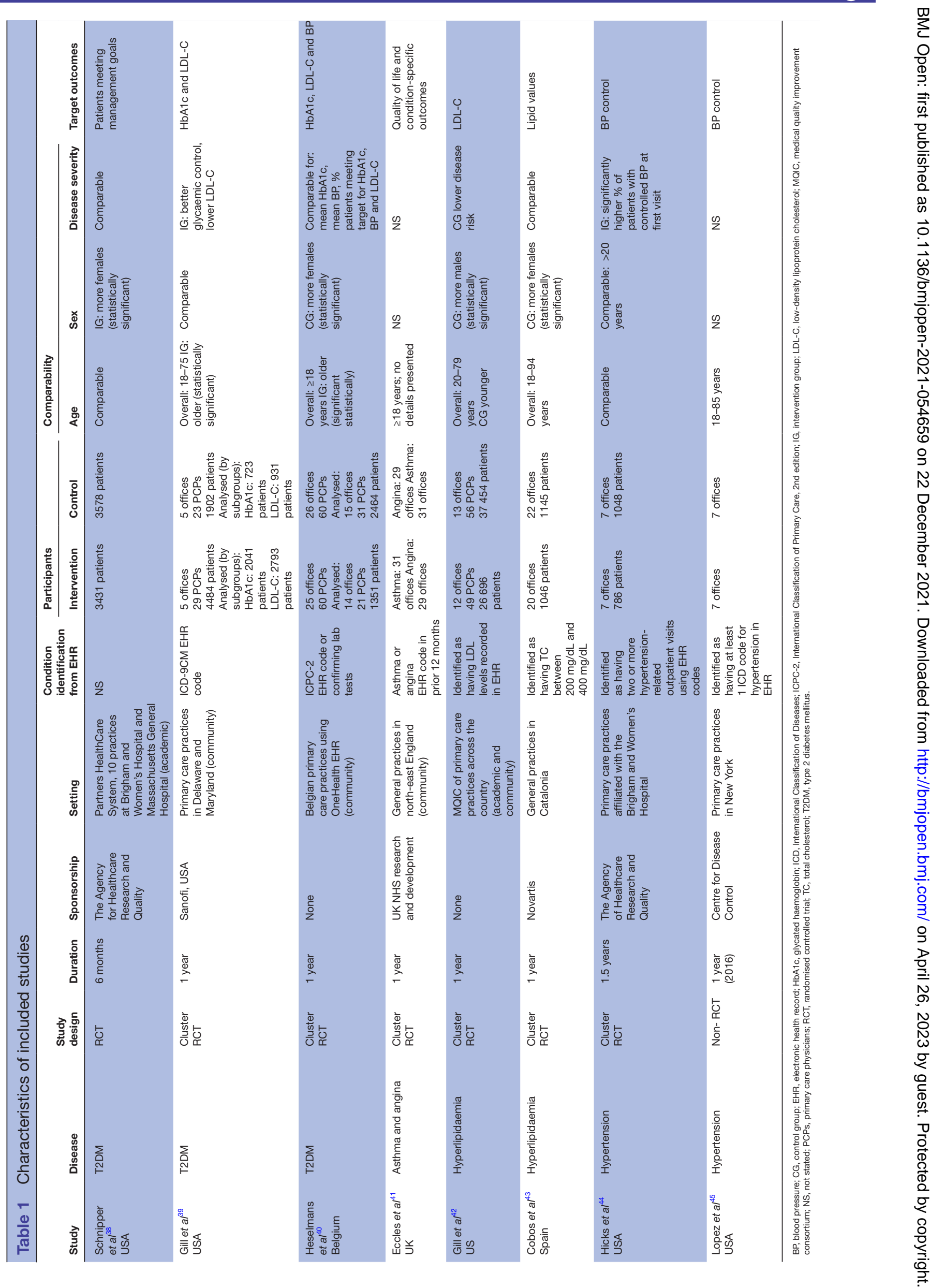


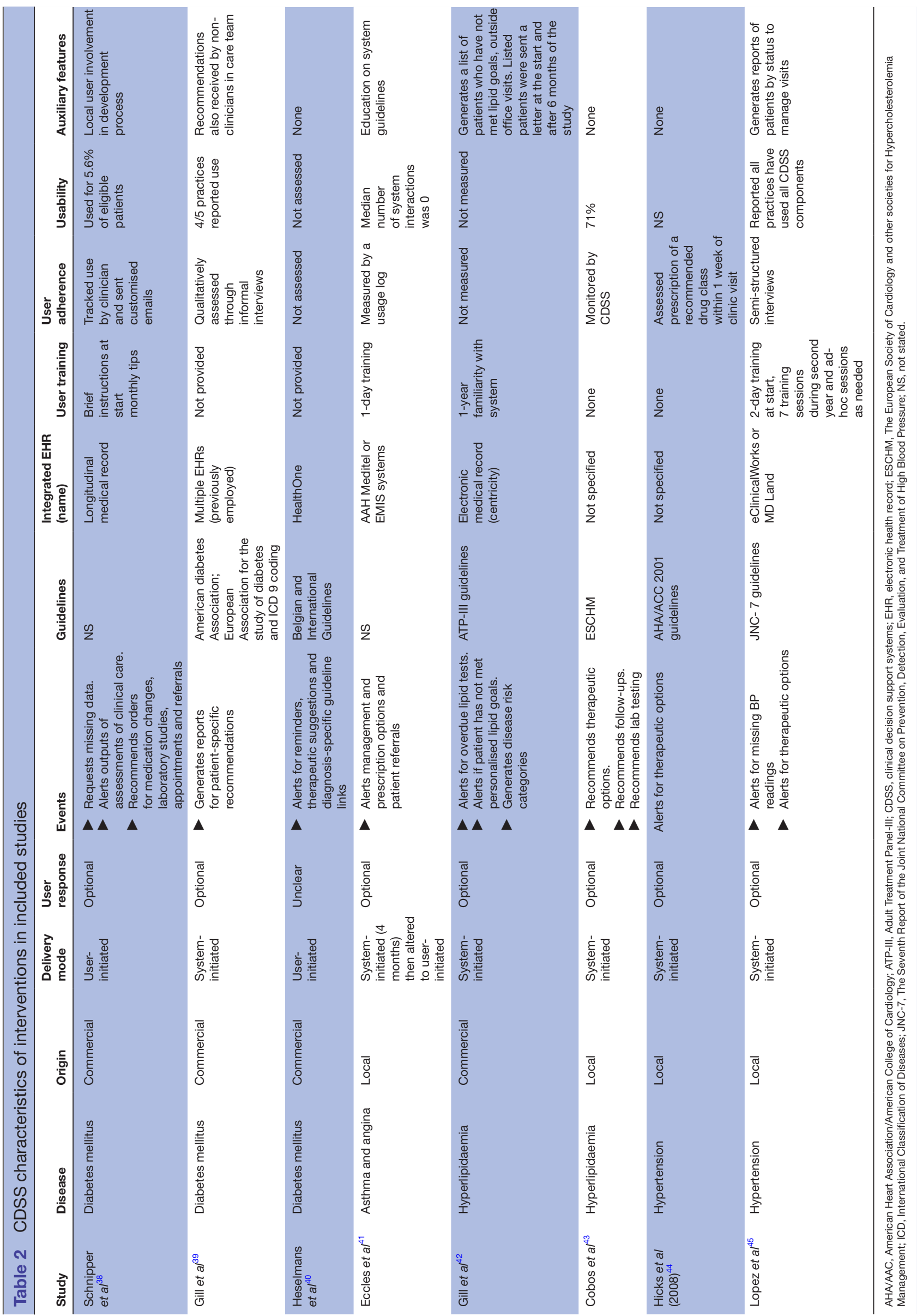




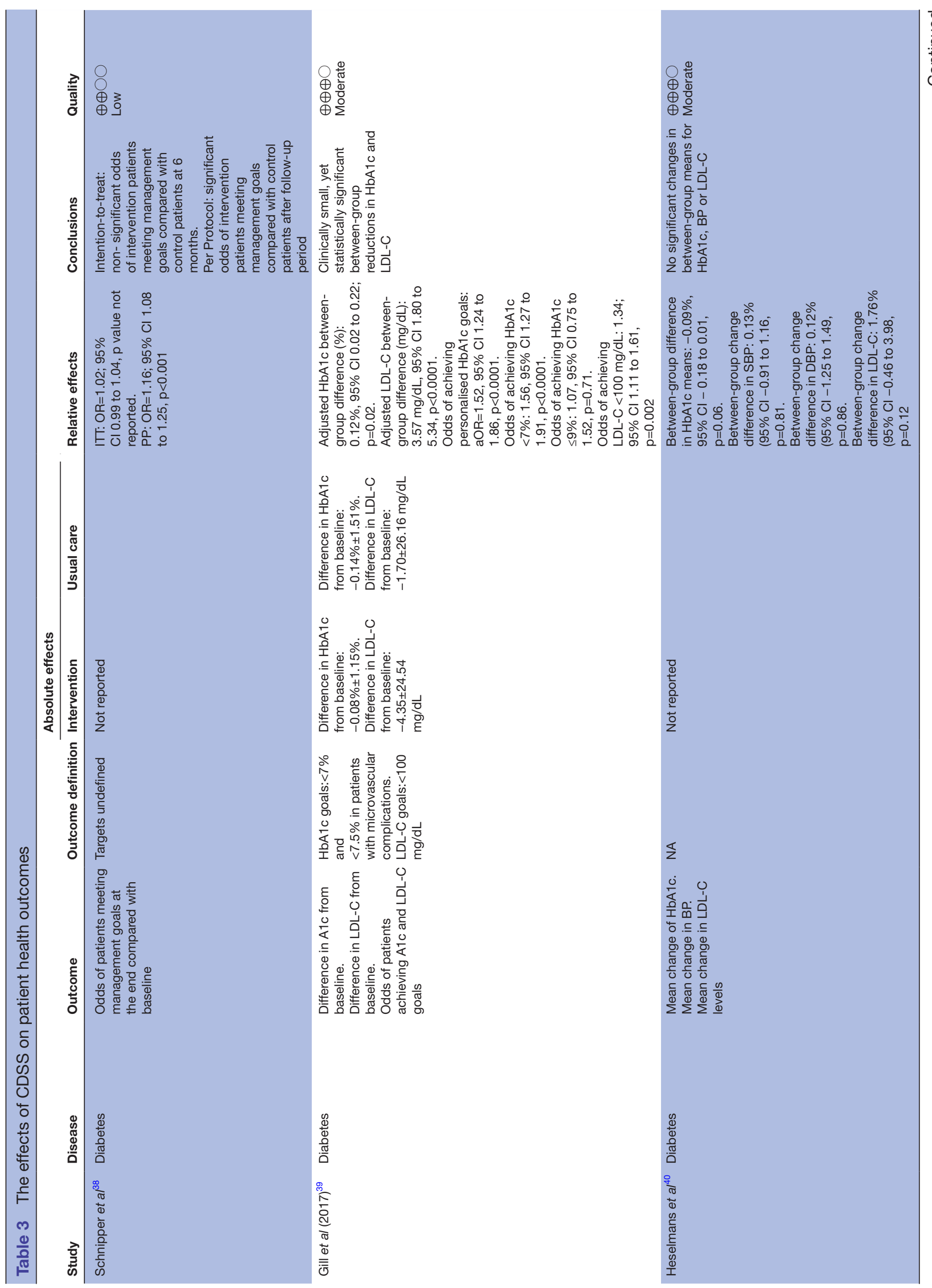




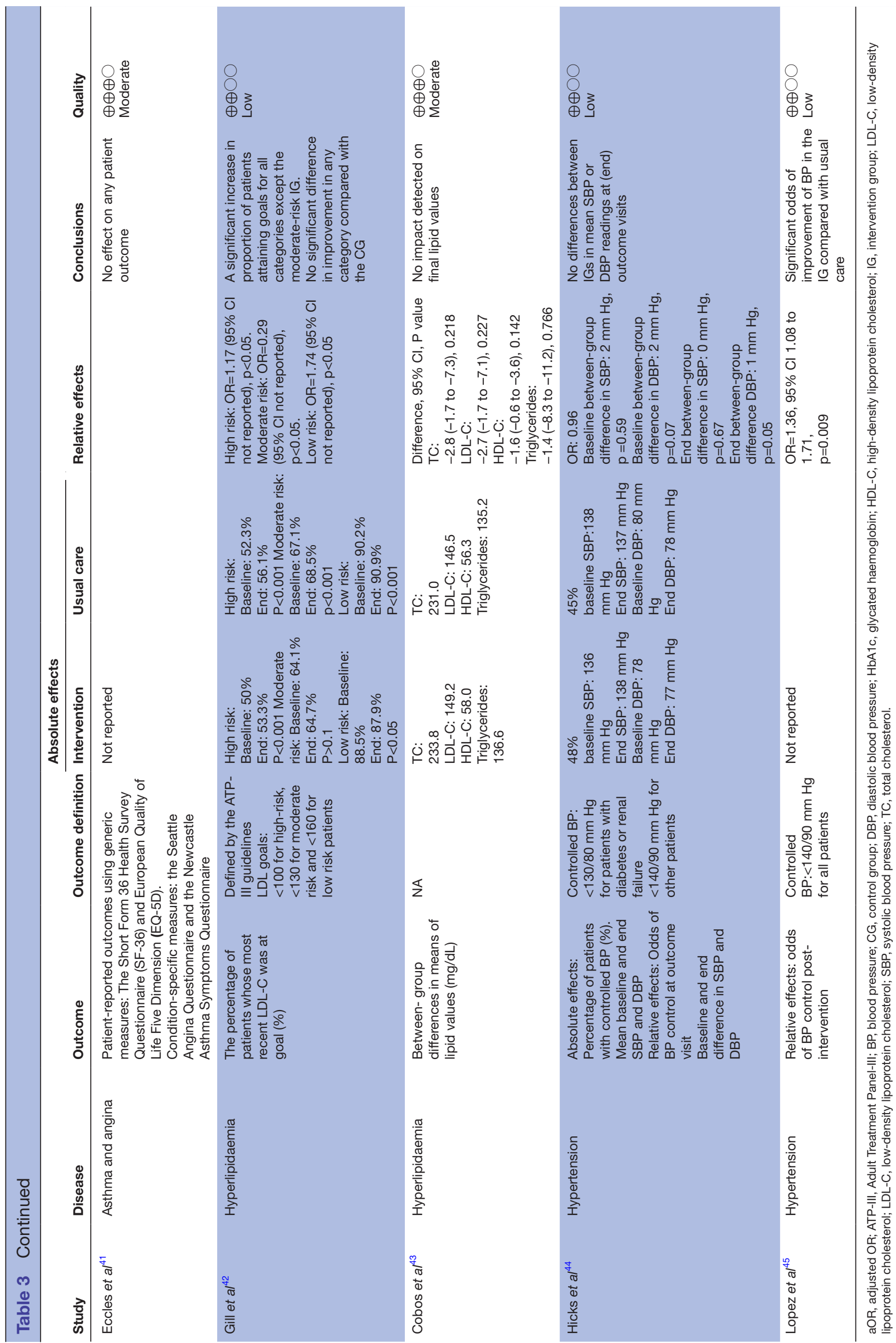


patients without missing HbA1c and LDL-C values were analysed (table 3 ).

Schnipper et al (RCT) reported the odds of patients meeting management goals (table 3 ) ${ }^{38}$ Intention-to-treat analysis showed non-significant changes in patients' odds of meeting management goals; a per-protocol analysis, however, showed slightly significant odds of attaining management goals in the intervention group compared with the control group (table 3). Among the three studies, this intervention is the only one that involved user training (table 2). Auxiliary features also included feedback on performance and local user (physician) involvement in the CDSS development process; yet, system usability was reported to be used for only $5.6 \%$ of patients (table 2 ).

Heselmans et al (cluster RCT) reported non-significant between-group differences in HbA1c, LDL-C, diastolic blood pressure (DBP) and systolic blood pressure (SBP) (table 3). ${ }^{40}$ Levels of HbAlc were relatively well controlled, with reasonable mean values for HbAlc in both groups at baseline, especially in the control group (table 1$).{ }^{40}$ Subsequent analysis of a subgroup of 601 patients with HbAlc $>7.0 \%$ retrieved a statistically significant between-group difference in HbAlc favouring the intervention, though not clinically relevant $(-0.40 \%$ (95\% CI -0.70 to -0.09$)$ ). The same analysis retrieved non-significant differences in LDL-C and BP. User adherence and usability were not measured.

In all three studies, there was no mention of the provision of reimbursement for the implementation of the CDSS. All CDSS were commercial, and user response to the system alerts was optional.

\section{Asthma and angina}

One study assessed the impact of CDSS on self-reported quality of life and condition-specific outcomes in patients with asthma and angina. ${ }^{41}$ The angina intervention group acted as the control group for the asthma intervention group and vice versa (justified by authors to eliminate a possible Hawthorne effect in practitioner behaviour on the primary outcome). Questionnaires were administered in three rounds (a year before the intervention, beginning of the intervention and a year after the intervention), which showed non-significant differences in both intervention groups post-intervention (table 3). Completion of the three rounds of questionnaires occurred in $46 \%$ and $35 \%$ of patients with angina and asthma, respectively. The implemented CDSS were locally produced and embedded in two widely used health systems (table 2). The CDSS were modified 4 months into the study from system to user-initiated, as requested by participating physicians. User training was limited, and system usability was very low, measured using a $\log$ with a median of zero usersystem interaction during most of the study (table 2).

Hyperlipidaemia

Two studies involved participants with hyperlipidaemia. $^{42} 43$
The study by Gill et al involved implementing a commercial CDSS in participating practices of one institution. ${ }^{42}$ All patients enlisted under participating practices with recorded LDL-C values were enrolled. A high correlation in outcomes was found at baseline and end measurements, causing patients not meeting LDL-C levels after randomisation to be analysed only. The control group also had a lower risk of disease and was significantly younger. Patients were stratified into three guideline-based risk groups, and the percentage of patients reaching the predefined goals at baseline and end were recorded (table 1). All categories in the intervention and control groups showed a significant increase in the proportion of patients attaining lipid goals, except in the moderaterisk group. Calculated differences were not presented; however, $p$ values of differences within the intervention and control groups suggested no significant improvement in any risk category.

In Cobos et al despite a usability of $71 \%$, the intervention had no superiority over usual care in terms of between-group differences in lipid values (table 3) ${ }^{43}$ The study reported $>25 \%$ patients lost to follow-up for clinic visits or LDL-C testing (table 1).

\section{Hypertension}

Two studies assessed the effect of CDSS on BP in hypertensive patients. ${ }^{44}$ Both studies employed CDSS that were system-initiated with optional user responses (table 2).

Hicks et al reported no differences between intervention groups in BP control (table 3). ${ }^{44}$ Differences in baseline and end SBP and DBP between groups were not significant. Nonetheless, the intervention group had a significantly higher percentage of patients with controlled BP (table 1). No user training was provided, and system usability was not measured (table 2). Less than $80 \%$ of recruited patients were followed-up due to withdrawals caused by the study's inclusion criteria, which required patients to have a recorded ethnicity in the EHR. Subsequently, patients without BP recordings were considered uncontrolled. However, a subset analysis excluding these patients still retrieved a non-significant difference in BP values.

Lopez et als study was a quasi-RCT and reported significant odds of BP improvement in the intervention group compared with the control group (table 3). ${ }^{45}$ Notably, extensive training was provided for participating clinicians compared with all other studies, and semi-structured interviews revealed that all practices appeared to use the system components (table 1 ).

\section{DISCUSSION}

\section{Main findings}

As primary care providers are increasingly required to monitor and manage chronic diseases, there is a need to assess the capabilities of CDSS on patient outcomes. This systematic review included the eight available studies on the effect of computerised, knowledge-based, 
EHR-embedded CDSS on clinical and patient-reported outcomes. Several study designs were included in the eligibility criteria; however, studies fit for inclusion were trials, namely, RCTs, cluster RCTs and non-RCTs (table 1, online supplemental table 1). Two studies reported a statistically significant impact of CDSS on patient outcomes, ${ }^{39} 45$ one reporting clinically small yet significant reductions in HbAlc and LDL-C and another reporting significant increases in the proportion of hypertensive patients who achieve goals, which may be clinically relevant. ${ }^{45}$ Another two studies reported statistically significant findings but in subgroup analyses. ${ }^{38} 40$ One study reported statistical significance in partial outcomes and three studies did not report any statistically significant impact of CDSS on outcomes at all. ${ }^{4-44}$ Overall, evidence was not conclusive to imply a differential effect, either positive or negative, of clinician use of CDSS on patient or clinical outcomes compared with usual care, mainly due to the quality of evidence impacted by the prominent RoB in the included studies. The systematic review also noted a limited availability of studies evaluating the CDSS of interest directed to clinicians in primary care for the management of the common chronic diseases. This is especially true for COPD, arthritis and osteoporosis, which were eligible conditions, yet no such studies were available.

\section{Implications of the review}

The systematic review infers the importance of considering how the interplay of factors such as the nature of a healthcare setting (academic and community) and CDSS interact with clinicians (or users per se). System-initiated CDSS seemed to have a better reflection on patient outcomes compared with user-initiated systems; however, this relationship was confounded by many factors such as poor process measures, namely, low system usability and EHR-CDSS interoperability difficulties. A common limitation reported across most studies was poor patient follow-up, which highlights the importance of patient behaviour as a confounding factor to the performance of CDSS interventions in clinical settings. Some interventions included helpful auxiliary features such as involvement of local physicians in CDSS development and feedback on performance; yet, these features did not seem to reflect positively on patient outcomes. Understanding user (clinician) experiences qualitatively in the context of using such CDSS to manage chronic diseases in primary care may be of benefit to inform on their practical use. This review assessed CDSS functionality when used by clinicians; however, CDSS accessibility to other members of a multidisciplinary team may have an added positive effect, which could translate into better patient outcomes. Multidisciplinary teams in primary care have been shown to aid successful implementation of CDSS by increasing adherence to CPGs and improving patient outcomes in the absence of CDSS. ${ }^{46} 47$

The healthcare setting and intervention implementation itself may dictate the success or failure of an intervention. Large academic care settings may be better equipped with IT and financial resources than community centres to ensure successful implementations of CDSS. ${ }^{48}$ Included studies revealed limitations due to poor implementation of the CDSS, namely, interoperability issues, lack of IT support, poor usability and lack of user training, which could have hindered the intervention from reflecting significant outcomes compared with usual care. Models have been offered to help explicate how structural elements of healthcare settings and various levels of healthcare organisations levelling up to the industry may influence clinician behaviour and uptake of health IT to influence patient outcomes. ${ }^{1749}$ Finally, it is imperative to consider the effect of non-clinical factors such as patient behaviour outside clinic visits, their perceptions and adherence to clinical advice. ${ }^{50}$

\section{Comparison with previous reviews}

Previous reviews of CDSS of multiple types have indicated impact on patient outcomes in some instances. A recent review showed that CDSS systems achieve small to moderate effects on processes of care; yet, the extent of translation to patient outcomes and under what clinical contexts and conditions this was achieved was not determined. ${ }^{24}$ Previous reviews were not limited to primary care settings and included various other CDSS, namely, paper-based, web-based, stand-alone electronic systems, and patient-directed systems that do not necessarily alert a clinician at the point of care ${ }^{192151}$ However, reviews assessing the impact of various types of CDSS on chronic disease patient outcomes in primary care identified inconclusive results, with a need to define more clearly the exact role of CDSS compared with usual care in subsequent studies. ${ }^{20}{ }^{23}$ Reviews evaluating patient outcomes noted conceptual between-study heterogeneity, as this review did, especially when several diseases and clinical outcomes were considered. ${ }^{52}$ In terms of patient outcomes, CDSS seem to affect clinical outcomes for cardiovascular risk factors such as hyperlipidaemia and diabetes. ${ }^{1153}$ Nevertheless, a much more recent review on the effect of CDSS on cardiovascular risks revealed no definitive clinical benefits after their implementation. ${ }^{53}$ On the other hand, the evidence is less robust for other non-cardiovascular risk-related chronic conditions such as cancer and other primary care sensitive cases (PCSCs) such as vaccination, which were not part of the eligibility criteria in this review. ${ }^{54}$

A review of CDSS embedded within EHRs in all-purpose healthcare settings on overall mortality and morbidity suggested no effect of CDSS on mortality. However, it noted that potential benefits or risks would highly depend on setting and disease characteristics, which is an evident implication in this review. ${ }^{52}$ A review by Bryan $e t$ $a l$ validated the use of CDSS in primary care to improve outcomes; however, they reported substantial variability in intervention effectiveness related to the type and implementation of the different types of CDSS. ${ }^{23}$ Additionally, other reviews incorporated studies that involved the use of CDSS by multidisciplinary care members, 
reflecting positive clinical outcomes with shared decisionmaking. ${ }^{195155}$

\section{Unanswered questions and future research}

More research is needed to establish the effectiveness of EHR-embedded CDSS on clinical outcomes of patients with chronic diseases treated in primary care settings. Further studies involving patients with chronic diseases are needed for robust evidence. Moreover, it may not be reasonable to evaluate the intervention's efficacy based on older studies, given the exponential growth and rapid advancements in the field of CDSS. ${ }^{56}$ Future research could include practitioner-related cost effectiveness and patient-related outcomes to present an ampler perspective on the effect of this type of CDSS to inform policy-making and clinicians. Other PCSCs can be studied for a broader scope, including cancer, mental health illnesses and preventative interventions such as vaccination.$^{57}$ Research around prolonged evaluations of CDSS is needed. Longer-term studies may account for potential implementation barriers that could take place. Additionally, it would be beneficial to synthesise findings on the effect of this particular type of CDSS on patient outcomes, when operated within a multidisciplinary team of non-clinician providers. It is worth noting that patient outcomes are not always addressed efficiently in trials, among the reasons is the possibility of having subjective, composite or surrogate outcomes, which do not necessarily translate into reallife patient outcome improvements. ${ }^{58}$ Hence, including observational studies in updated reviews may be useful. Additionally, no studies of registries embedded with CDSS fit the eligibility criteria, suggesting a potentially greater inclination to embed CDSS with EHRs currently. There also seems to be a dearth of studies evaluating non-cardiovascular risk-related chronic diseases postimplementation of CDSS linked with EHRs, as no studies evaluating patients with COPD, osteoporosis and arthritis were eligible.

\section{Strengths and limitations}

This systematic review focuses on interventions involving a specific type of CDSS - those that are knowledge-based, coupled with EHR systems, that provide electronic alerts and are used by physicians in primary care to support chronic disease management. To the authors' knowledge, no such review exists considering this combined focus and given the attention these systems are increasingly gaining in primary care settings. ${ }^{29}$ As an example of the novelty of this review, compared with previous reviews with the most similar focus to our own by Bryan and Boren ${ }^{23}$ and Bright et al ${ }^{19}$ this review included studies not featured in these previous reviews such as the studies by Schnipper et al, Gill et al, Heselmans et $a l$ and Hicks et al. ${ }^{38-40}{ }^{44}$ Application of the eligibility criteria identified eight studies for inclusion, which may be a small number given the recent growth of interest in CDSS in healthcare settings. ${ }^{59}$ Nevertheless, piloting the electronic search strategy and supplementing it with hand searching of reference lists of previous reviews in the field permitted a sufficient level of confidence that all relevant articles, against the eligibility criteria, were included for synthesis. The review also contributes to the knowledge provided by a recent review that focuses on process outcomes. ${ }^{24}$

Some limitations arose from methodological constraints. Studies were of an average of 1-year duration, which makes it difficult to deduce long-term effects on patient outcomes. Nine common chronic diseases were included in the syntax. ${ }^{28}$ However, there was an absence of eligible studies in five of them, which limited the number of studies for inclusion. Although a selection of nine chronic diseases was included in the syntax, these covered the most common chronic diseases, as done by Reynold $e t a l .{ }^{28}$ The review could have covered other PCSCs for a broader scope, including cancer and mental health illnesses, per se. The included studies were also of low-to-moderate quality of evidence. Additionally, our review does not report on process outcomes. The decision to exclude process outcomes such as practitioner-related outcomes, namely, clinician adherence to CPGs was made to channel the research question to focus on where the most significant knowledge gap lies, as the effect of CDSS on patient outcomes remains largely uncertain. ${ }^{19-21} 2324$ Nevertheless, capturing those outcomes would have provided a more comprehensive assessment on the effect of CDSS for the management of patients with chronic disease in primary care. The heterogeneity in study intervention components, participant covariates, setting attributes and CDSS characteristics rendered outcome pooling in a meta-analysis not suitable.

\section{Conclusion}

This systematic review aimed to assess the impact of CDSS integrated with EHRs on patient-reported and clinical outcomes of chronic disease patients in primary care settings. A definite conclusion on the intervention effect on outcomes could not be drawn due to betweenstudy heterogeneity and methodological risks, reflecting an immature research area. Intervention characteristics and implementation approaches may have an impact on outcomes from CDSS use and we make reference to the literature in this area that may guide and help evaluate successful implementations of CDSS in practice. Further studies are needed to formulate more robust inferences and validate the intervention's effect on patient outcomes. With the continuous rise in the prevalence of chronic diseases, it is essential to understand how enhancing primary care management using IT support could support improved outcomes. Practical applications of CDSS evaluations in the healthcare workflow and setting should be sustained to suitably determine the actual effectiveness of CDSS and support improved implementation efforts into practice. Strengthening the clinical benefit of CDSS and determining their cost 
effectiveness could inform policy-making of enhanced primary healthcare of chronic disease management.

\section{Author affiliations}

${ }^{1}$ Self Care Academic Research Unit, Imperial College London, London, UK

${ }^{2}$ Department of Primary Care and Public Health, Imperial Centre for Cardiovascular Disease Prevention, Imperial College London, London, UK

${ }^{3}$ Imperial Centre for Cardiovascular Disease Prevention (ICCP), Department of Primary Care and Public Health, School of Public Health, Imperial College London. London, United Kingdom, London, UK

${ }^{4}$ Department of Medicine, Faculty of Medicine, University of Seville, Seville, Spain ${ }^{5}$ Clinical Epidemiology and Vascular Risk, Instituto de Biomedicina de Sevilla, IBiS/ Hospital Universitario Virgen del Rocío/Universidad de Sevilla/CSIC, Seville, Spain ${ }^{6}$ Department of Primary Care Clinical Sciences, Institute of Applied Health Research, University of Birmingham, Birmingham, UK

${ }^{7}$ Department of Primary Care and Public Health, School of Public Health, Imperial College London, London, UK

Acknowledgements The authors would like to thank the library services team at Imperial College London. Special thanks to Rebecca Jones for her support in reviewing the search strategy. KID acknowledges support from a PhD studentship from the National Institute for Health Research (NIHR) under the Applied Health Research (ARC) programme for North West London, UK (the views expressed in this publication are those of the authors and not necessarily those of the NHS, the NIHR or the Department of Health). AJV-V acknowledges support from a fellowship from the 'Programa Beatriz Galindo' from the Ministry of Universities of Spain, and University of Seville, Spain.

Contributors Rl conceived the original study concept with input from NM. NM and AJV-V led the overall study design and supervised the subsequent steps of the study. MIEA and KID designed the search strategy with supervision from the other authors and conducted the literature search, data extraction and quality assessment of studies. MLEA wrote the first draft of the manuscript. All authors participated in the interpretation of data, have critically reviewed the manuscript and provided edits and comments, and approved its final submission. NM acts as the guarantor and RI and NM are joint principal/last authors.

Funding The authors have not declared a specific grant for this research from any funding agency in the public, commercial or not-for-profit sectors.

Competing interests RI and NM are employees of Cerner UK, a health technology organisation. AJV-V reports past or current participation in investigator-initiated research grants to Imperial College London from Pfizer, Amgen, Sanofi, MSD, Daiichi Sankyo and Regeneron, honoraria for lectures from Amgen, Mylan and Akcea, and steering committee/consulting fees from Radcliffe Cardiology and Bayer, all outside the submitted work. KID reports past or current participation in investigator-initiated research grants to Imperial College London from Amgen, Sanofi, MSD, Daiichi Sankyo and Regeneron and consulting fees from Bayer, all outside the submitted work.

\section{Patient consent for publication Not applicable.}

Ethics approval This study does not involve human participants. The present work relied on already published aggregated data and ethics committee approval was unnecessary.

Provenance and peer review Not commissioned; externally peer reviewed.

Data availability statement Data sharing not applicable as no datasets generated and/or analysed for this study. The present work relied on already published aggregated data.

Supplemental material This content has been supplied by the author(s). It has not been vetted by BMJ Publishing Group Limited (BMJ) and may not have been peer-reviewed. Any opinions or recommendations discussed are solely those of the author(s) and are not endorsed by BMJ. BMJ disclaims all liability and responsibility arising from any reliance placed on the content. Where the content includes any translated material, BMJ does not warrant the accuracy and reliability of the translations (including but not limited to local regulations, clinical guidelines, terminology, drug names and drug dosages), and is not responsible for any error and/or omissions arising from translation and adaptation or otherwise.

Open access This is an open access article distributed in accordance with the Creative Commons Attribution Non Commercial (CC BY-NC 4.0) license, which permits others to distribute, remix, adapt, build upon this work non-commercially, and license their derivative works on different terms, provided the original work is properly cited, appropriate credit is given, any changes made indicated, and the use is non-commercial. See: http://creativecommons.org/licenses/by-nc/4.0/.

\section{ORCID iD}

Marie line El Asmar http://orcid.org/0000-0002-8481-6431

\section{REFERENCES}

1 Viswanathan M, Golin CE, Jones CD, et al. Interventions to improve adherence to self-administered medications for chronic diseases in the United States: a systematic review. Ann Intern Med 2012;157:785-95.

2 CDC. Health and economic costs of chronic diseases. Available: https://www.cdc.gov/chronicdisease/about/costs/index.htm

3 van Oostrom SH, Picavet HSJ, de Bruin SR, et al. Multimorbidity of chronic diseases and health care utilization in general practice. BMC Fam Pract 2014;15:61.

4 Lehnert T, Heider D, Leicht $\mathrm{H}$, et al. Review: health care utilization and costs of elderly persons with multiple chronic conditions. Med Care Res Rev 2011;68:387-420.

5 WHO. Innovative care for chronic conditions: building blocks for action. Available: http://www.who.int/chp/knowledge/publications/ icccreport/en/

6 Francesconi P, Ballo P, Profili F. Chronic care model for the management of patients with heart failure in primary care. Health Serv Insights 2019;12:117863291986620.

7 Stellefson M, Dipnarine K, Stopka C. The chronic care model and diabetes management in US primary care settings: a systematic review. Prev Chronic Dis 2013;10:E26.

8 Oprea L, Cojocaru D, Sandu A. The chronic care model (CCM) and the social gradient in health. Revista de Cercetare si Interventie Sociala 20132013;41:176--89.

9 Murad $\mathrm{MH}$. Clinical practice guidelines: a primer on development and dissemination. Mayo Clin Proc 2017;92:423-33.

10 Fürthauer J, Flamm M, Sönnichsen A. Patient and physician related factors of adherence to evidence based guidelines in diabetes mellitus type 2, cardiovascular disease and prevention: a cross sectional study. BMC Fam Pract 2013;14:47.

11 Nichol MB, Knight TK, Priest JL, et al. Nonadherence to clinical practice guidelines and medications for multiple chronic conditions in a California Medicaid population. J Am Pharm Assoc 2010;50:496-507.

12 Wasylewicz ATM. Scheepers-Hoeks, clinical decision support systems. Fundamental Clin Data Sci 2018.

13 Jao C. Efficient decision support systems: practice and challenges in biomedical related domain. BoD-Books on Demand 2011.

14 Haynes RB, Wilczynski NL, Computerized Clinical Decision Support System (CCDSS) Systematic Review Team. Effects of computerized clinical decision support systems on practitioner performance and patient outcomes: methods of a decision-maker-researcher partnership systematic review. Implement Sci 2010;5:12.

15 Sutton RT, Pincock D, Baumgart DC, et al. An overview of clinical decision support systems: benefits, risks, and strategies for success. NPJ Digit Med 2020;3:17.

16 Shortliffe EH, Shortliffe EH, Cimino JJ. Biomedical informatics: computer applications in health care and biomedicine. Springer, 2014.

17 Kaushal JSA, Alison E, Sarah N. Effects of workload, work complexity, and repeated alerts on alert fatigue in a clinical decision support system. BMC Med Informat Decision Making 2017;17:1-9.

18 Shahsavarani AM. Clinical decision support systems (CDSS): state of the art review of literature. Int J Med Rev 2015;2:299-308.

19 Bright TJ, Wong A, Dhurjati R, et al. Effect of clinical decision-support systems: a systematic review. Ann Intern Med 2012;157:29-43.

20 Souza NM, Sebaldt RJ, Mackay JA, et al. Computerized clinical decision support systems for primary preventive care: a decisionmaker-researcher partnership systematic review of effects on process of care and patient outcomes. Implement Sci 2011;6:87.

21 Garg AX, Adhikari NKJ, McDonald H, et al. Effects of computerized clinical decision support systems on practitioner performance and patient outcomes: a systematic review. JAMA 2005;293:1223-38.

22 Lobach D, Sanders GD, Bright TJ. Methods: Agency for Healthcare Research and Quality (US) 2012.

23 Bryan C, Boren SA. The use and effectiveness of electronic clinical decision support tools in the ambulatory/primary care setting: a systematic review of the literature. Inform Prim Care 2008;16:79-91.

24 Kwan JL, Lo L, Ferguson J, et al. Computerised clinical decision support systems and absolute improvements in care: meta-analysis of controlled clinical trials. BMJ 2020;370:m3216. 
25 Moher D, Liberati A, Tetzlaff J, et al. Preferred reporting items for systematic reviews and meta-analyses: the PRISMA statement. PLoS Med 2009;6:e1000097.

26 Liberati A, Altman DG, Tetzlaff J, et al. The PRISMA statement for reporting systematic reviews and meta-analyses of studies that evaluate healthcare interventions: explanation and elaboration. $B M J$ 2009;339:b2700.

27 Howard C. Subject and course guides: evidence based medicine: PICO. Available: https://researchguides.uic.edu/c.php?g=252338\&p= 3954402

28 Reynolds R, Dennis S, Hasan I, et al. A systematic review of chronic disease management interventions in primary care. BMC Fam Pract 2018;19:1-3.

29 Jansma EP, Vries RD. Patient reported outcome measures (PROMs). bmi-online search blocks. Available: https://blocks.bmi-online.nl/ catalog/248

30 Data extraction forms. Available: https://dplp.cochrane.org/dataextraction-forms

31 Lobach D, Sanders GD, Bright TJ. Data abstraction guidance. Agency for Healthcare Research and Quality (US), 2012.

32 Zhao KF, Kelsey Y, Matthew CT. Risk of bias tools in systematic reviews of health interventions: an analysis of PROSPERO-registered protocols. System Rev 2019;8:1-9.

33 Higgins JPT, Altman DG, Gøtzsche PC, et al. The Cochrane collaboration's tool for assessing risk of bias in randomised trials. BMJ 2011;343:d5928.

34 Higgins JP, Savović J, Page MJ, et al. Assessing risk of bias in a randomized trial. Cochrane Handbook Syst Rev Intervent 2019:23:205-28.

35 Ryan R, Hill S, Prictor M. Cochrane consumers \& communication review group study quality guide, guide for review authors on assessing study quality, 2013.

36 BMJ Best Practice. What is GRADE? Available: https://bestpractice. bmj.com/info/toolkit/learn-ebm/what-is-grade/

37 Meader N, King K, Llewellyn A, et al. A checklist designed to aid consistency and reproducibility of GRADE assessments: development and pilot validation. Syst Rev 2014;3:82.

38 Schnipper JL, Linder JA, Palchuk MB, et al. Effects of documentation-based decision support on chronic disease management. Am J Manag Care 2010;16:SP72-81.

39 Gill J, Kucharski K, Turk B, et al. Using electronic clinical decision support in patient-centered medical homes to improve management of diabetes in primary care: the decide study. J Ambul Care Manage 2019;42:105-15.

40 Heselmans A, Delvaux N, Laenen A, et al. Computerized clinical decision support system for diabetes in primary care does not improve quality of care: a cluster-randomized controlled trial. Implement Sci 2020;15:5.

41 Eccles M, McColl E, Steen N. Computerised evidence-based guidelines may not improve asthma or angina management in primary care. Evidence-Based Healthcare 2003;7:81-2.

42 Gill JM, Chen YX, Glutting JJ, et al. Impact of decision support in electronic medical records on lipid management in primary care. Popul Health Manag 2009;12:221-6.

43 Cobos A, Vilaseca J, Asenjo C. Cost effectiveness of a clinical decision support system based on the recommendations of the European Society of Cardiology and other societies for the management of hypercholesterolemia. Disease Manage Health Outcome 2005;13:421-32.
44 Hicks LS, Sequist TD, Ayanian JZ, et al. Impact of computerized decision support on blood pressure management and control: a randomized controlled trial. J Gen Intern Med 2008;23:429-41.

45 Lopez PM, Divney A, Goldfeld K, et al. Feasibility and outcomes of an electronic health record intervention to improve hypertension management in Immigrant-serving primary care practices. Med Care 2019:57:S164-71.

46 Goud R, de Keizer NF, ter Riet G, et al. Effect of guideline based computerised decision support on decision making of multidisciplinary teams: cluster randomised trial in cardiac rehabilitation. BMJ 2009;338:b1440.

47 Manns BJ, Tonelli M, Zhang J, et al. Enrolment in primary care networks: impact on outcomes and processes of care for patients with diabetes. Can Med Assoc J 2012;184:E144-52.

48 McClellan SR, Casalino LP, Shortell SM, et al. When does adoption of health information technology by physician practices lead to use by physicians within the practice? J Am Med Inform Assoc 2013;20:e26-32.

49 Latoszek-Berendsen A, Tange H, van den Herik HJ, et al. From clinical practice guidelines to computer-interpretable guidelines. A literature overview. Methods Inf Med 2010;49:550-70.

50 Hajiaj FM, Salek MS, Basra MKA, et al. Non-clinical influences on clinical decision-making: a major challenge to evidence-based practice. J R Soc Med 2010;103:178-87.

51 Varghese J, Kleine M, Gessner SI, et al. Effects of computerized decision support system implementations on patient outcomes in inpatient care: a systematic review. J Am Med Inform Assoc 2018;25:593-602.

52 Moja L, Kwag KH, Lytras T, et al. Effectiveness of computerized decision support systems linked to electronic health records: a systematic review and meta-analysis. Am J Public Health 2014:104:e12-22. doi:10.2105/AJPH.2014.302164

53 Groenhof TKJ, Asselbergs FW, Groenwold RHH, et al. The effect of computerized decision support systems on cardiovascular risk factors: a systematic review and meta-analysis. BMC Med Inform Decis Mak 2019;19:108.

54 Roshanov PS, Misra S, Gerstein HC, et al. Computerized clinical decision support systems for chronic disease management: a decision-maker-researcher partnership systematic review. Implement Sci 2011;6:92.

55 Kooij L, Groen WG, van Harten WH. The effectiveness of information Technology-Supported shared care for patients with chronic disease: a systematic review. J Med Internet Res 2017;19:e221.

56 Castaneda C, Nalley K, Mannion C, et al. Clinical decision support systems for improving diagnostic accuracy and achieving precision medicine. J Clin Bioinforma 2015;5:4.

57 Gibbons RJ, Chatterjee K, Daley J, et al. ACC/AHA/ACP-ASIM guidelines for the management of patients with chronic stable angina: a report of the American College of Cardiology/American heart association Task force on practice guidelines (Committee on management of patients with chronic stable angina). J Am Coll Cardiol 1999;33:2092-197.

58 Heneghan C, Goldacre B, Mahtani KR. Why clinical trial outcomes fail to translate into benefits for patients. Trials 2017;18:1-7.

59 Afzal M, Hussain M, Ali T, et al. Knowledge-based query construction using the CDSS knowledge base for efficient evidence retrieval. Sensors 2015;15:21294-314. 